\title{
Vascular endothelial growth factor-C ameliorates renal interstitial fibrosis through lymphangiogenesis in mouse unilateral ureteral obstruction
}

\author{
Shoko Hasegawa', Toshiaki Nakano ${ }^{1}$, Kumiko Torisu', Akihiro Tsuchimoto', Masahiro Eriguchi', Naoki Haruyama', \\ Kosuke Masutani ${ }^{1}$, Kazuhiko Tsuruya ${ }^{1,2}$ and Takanari Kitazono ${ }^{1}$
}

Renal fibrosis is the final common pathway of chronic kidney diseases. Lymphatic vessel (LV) proliferation is found in human renal diseases and other fibrotic diseases, suggesting that lymphangiogenesis is associated with the progression or suppression of kidney diseases. However, the purpose of LV proliferation is not completely understood. We investigated the effect of vascular endothelial growth factor (VEGF)-C on lymphangiogenesis, inflammation, and fibrosis in the mouse kidney using the unilateral ureteral obstruction (UUO) model. In UUO mice, significant proliferation of LVs was accompanied by tubulointerstitial nephritis and fibrosis. We continuously administered recombinant human VEGF-C to UUO model mice using an osmotic pump (UUO+VEGF-C group). Lymphangiogenesis was significantly induced in the UUO + VEGF-C group compared with the vehicle group, despite similar numbers of capillaries in both groups. The number of infiltrating macrophages, and levels of inflammatory cytokines and transforming growth factor- $\beta 1$ were reduced in the UUO+VEGF-C group compared with the vehicle group. Renal fibrosis was consequently attenuated in the UUO+VEGF-C group. In cultured lymphatic endothelial cells, administration of VEGF-C increased the activity and proliferation of lymphatic endothelial cells (LECs) and expression of adhesion molecules such as vascular cell adhesion molecule-1. These findings suggest that induction of lymphangiogenesis ameliorates inflammation and fibrosis in the renal interstitium. Enhancement of the VEGF-C signaling pathway in LECs may be a therapeutic strategy for renal fibrosis.

Laboratory Investigation (2017) 97, 1439-1452; doi:10.1038/labinvest.2017.77; published online 30 October 2017

Renal fibrosis is the final common pathway of chronic kidney diseases (CKDs). However, the pathological mechanism remains unclear. An increase in the number of lymphatic vessels (LVs; referred to as lymphangiogenesis) is found at the site of tubulointerstitial lesions and correlates with the degree of tissue damage in human renal diseases. ${ }^{1}$ Lymphangiogenesis is active in adult tissues under various pathological conditions such as inflammation, ${ }^{2,3}$ obesity, ${ }^{4}$ hypertension, ${ }^{5}$ lymphedema, ${ }^{6}$ and pulmonary fibrosis. ${ }^{7}$ However, the purpose of LV proliferation is not completely understood. We have previously reported that a high perivascular lymphatic density in kidney transplant patients is associated with the kidney allograft prognosis. ${ }^{8}$

The best known factors to promote lymphangiogenesis are vascular endothelial growth factor (VEGF)-C and VEGF-D. VEGF-C binds to VEGF receptor-2 (VEGFR-2) and VEGFR-3, which plays a crucial role in proliferation, migration, and survival of lymphatic endothelial cells (LECs) via VEGFR-3, ${ }^{9}$ and has lesser effects on blood vessels via VEGFR-2. ${ }^{10-12}$ VEGF$\mathrm{C}$ deficiency results in embryonic death of mice. ${ }^{13}$ Keratin 14/VEGF-C transgenic mice have an expanded network of cutaneous LVs. Transgenic expression of VEGF-C significantly limits acute skin inflammation in two experimental models with strong reduction of dermal edema. ${ }^{2}$ VEGFR-3 is a receptor of VEGF-C and mainly expressed in LECs of adult tissues. ${ }^{14}$ In C57BL/6J limb salvage model mice, Onimaru et al. ${ }^{15}$ revealed that blocking of VEGFR-3 induces marked swelling of hindlimbs and reduces their limb salvage score. The VEGF-C and VEGFR-3 signaling pathway is predicted to be important in tissue inflammation that is accompanied by swelling and edema.

Two of the main functions of LVs are to enforce the balance of interstitial fluid transport and maintain tissue

\footnotetext{
${ }^{1}$ Department of Medicine and Clinical Science, Graduate School of Medical Sciences, Kyushu University, Fukuoka, Japan and ${ }^{2}$ Department of Integrated Therapy for Chronic Kidney Disease, Graduate School of Medical Sciences, Kyushu University, Fukuoka, Japan

Correspondence: Dr K Tsuruya, MD, PhD, Department of Integrated Therapy for Chronic Kidney Disease, Graduate School of Medical Sciences, Kyushu University, 3-1-1 Maidashi, Higashi-ku, Fukuoka 812-8582, Japan.

E-mail: tsuruya@intmed2.med.kyushu-u.ac.jp

Received 11 January 2017; revised 30 May 2017; accepted 16 June 2017
} 
homeostasis. ${ }^{16,17}$ In almost all CKDs, renal interstitial swelling and edema are common findings. Such swelling of the renal interstitial space is known to induce remodeling of the interstitial matrix and renal fibrosis as a consequence. Because CKDs are multifactorial diseases with complex pathophysiologies, renal edema is considered as one of the active factors in

a

Control group (sham-operated)

$$
\mathrm{n}=8
$$

UUO+PBS group

$\mathrm{n}=8 / / \mathrm{PBS} 6.4 \mu \mathrm{L} / \mathrm{day}$ infusion by micro-osmotic pump

UUO+VEGF-C group

$\mathrm{n}=8$ 册 VEGF-C $10 \mu \mathrm{g} / 100 \mathrm{gBW} /$ day infusion by micro-osmotic pump
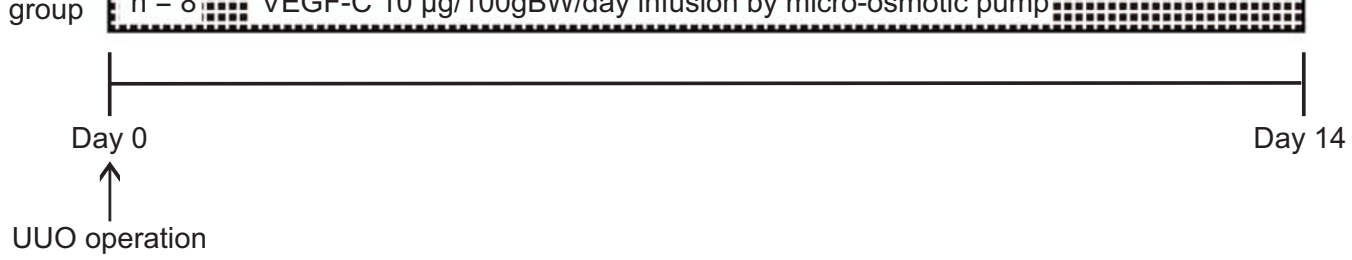

b

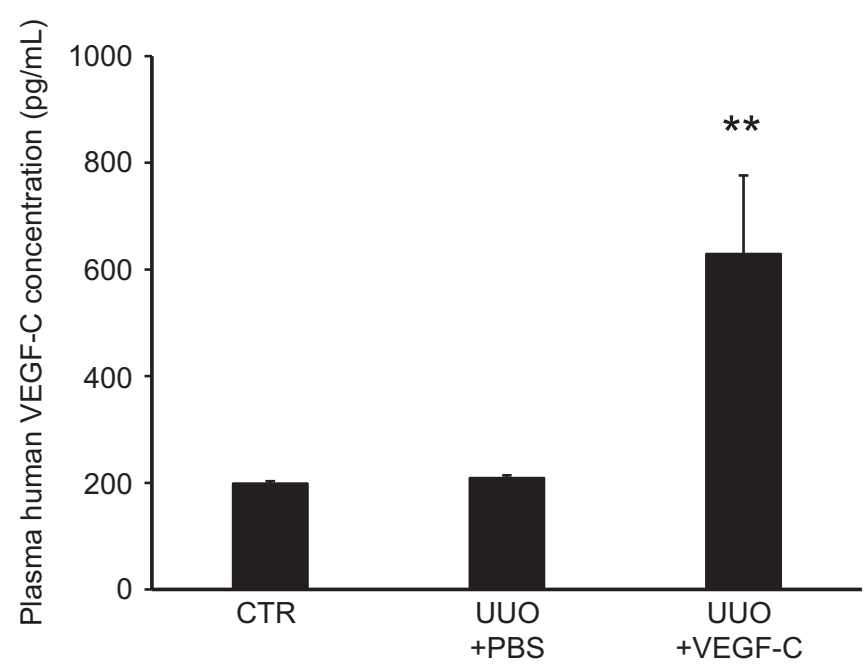

C
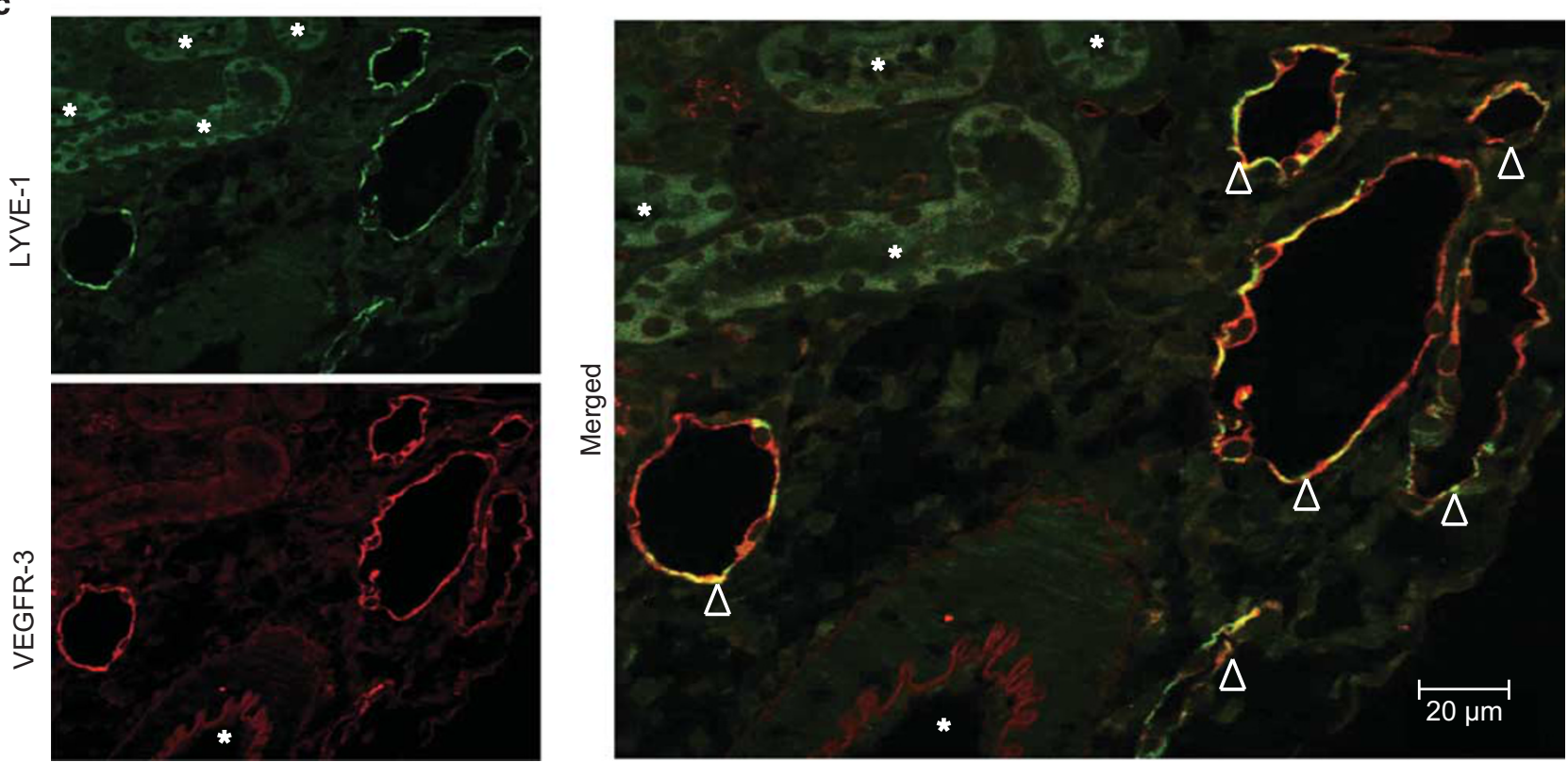
promoting CKD. The potential significance of LVs can be evaluated by modulating their development in experimental models of CKD. Furthermore, many renal diseases are associated with inflammatory reactions. Therefore, targeting LVs might be beneficial to reduce the progression of renal inflammation.

In this study, we hypothesized that enhancement of the VEGF-C/VEGFR-3 signaling pathway may attenuate renal fibrosis via improvement of interstitial inflammation. We investigated the effect of VEGF-C on lymphangiogenesis, inflammation, and fibrosis in the kidneys of unilateral ureteral obstruction (UUO) mice, a model of interstitial inflammation and fibrosis. Furthermore, we investigated the effect of VEGF$\mathrm{C}$ on cell viability, proliferation, and expression of adhesion molecules in LECs in vitro.

\section{MATERIALS AND METHODS In Vivo Experiments}

All experimental protocols were approved by the Ethics Committee on Animal Experimentation, Kyushu University Graduate School of Medical Sciences (approval number, A28229-0). Seven-week-old male C57BL/6J mice (Kyudo, Tosu, Japan) were individually housed in a specific pathogen-free environment with a 12-h day/night cycle and allowed free access to water. Experimental unilateral ureteral ligation resulting in UUO was performed (day 0) in 7-week-old C57BL/6J mice by ligation of the left ureter of each animal at the ureteropelvic junction after intraperitoneal injection of $0.3 \mathrm{mg} / \mathrm{kg}$ medetomidine hydrochloride, $4 \mathrm{mg} / \mathrm{kg}$ midazolam, and $5 \mathrm{mg} / \mathrm{kg}$ butorphanol tartrate, and inhalation of sevoflurane. To explore the effect of VEGF-C administration on renal lymphangiogenesis, inflammation, and fibrosis, C57BL/ $6 \mathrm{~J} \mathrm{male} \mathrm{mice} \mathrm{were} \mathrm{divided} \mathrm{into} \mathrm{three} \mathrm{groups:} \mathrm{control,} \mathrm{UUO}$ + PBS, and UUO+VEGF-C (Figure 1a). For the UUO+PBS group, $\mathrm{PBS}$ was administered to mice at $6.4 \mu \mathrm{l}$ per day using an intraperitoneal micro-osmotic pump (1002-0004317, Alzet, Cupertino, CA, USA) starting at day 0 and ending at day 14. For the VEGF-C-treated group, recombinant human VEGF-C (752-VC-025, R\&D Systems, Minneapolis, MN, USA) dissolved in the same volume of PBS was administered to mice at $10 \mu \mathrm{g} / 100 \mathrm{~g}$ per day using the intraperitoneal micro-osmotic pump starting at day 0 and ending at day 14 . We used recombinant human VEGF-C (Cys156Ser), a selective VEGFR-3 agonist to VEGF-C. ${ }^{18}$ The applied dose of VEGF-C has been used for VEGF-A to promote renal angiogenesis $^{19}$ and a higher dose $(200 \mathrm{ng} / \mathrm{g})$ of VEGF-C enhances VEGFR-3 phosphorylation within $30 \mathrm{~min}$ in vivo. ${ }^{20}$

\section{Sample Collection}

Mice were killed on day 14 ( $n=8$ each) by intraperitoneal injection of $0.3 \mathrm{mg} / \mathrm{kg}$ medetomidine hydrochloride, $4 \mathrm{mg} / \mathrm{kg}$ midazolam, and $5 \mathrm{mg} / \mathrm{kg}$ butorphanol tartrate. Blood samples were collected from the inferior vena cava, aliquoted for later analysis, and stored at $-80^{\circ} \mathrm{C}$. Immediately after blood collection, $50 \mathrm{ml}$ ice-cold $\mathrm{PBS}$ ( $\mathrm{pH}$ 7.4) was slowly perfused to harvest both kidneys. Renal samples were snap-frozen in liquid nitrogen and stored at $-80^{\circ} \mathrm{C}$.

\section{Histological Examination}

Samples from both kidneys were fixed in neutral-buffered 10\% formalin (062-01661, Wako Pure Chemical Industries, Osaka, Japan) and embedded in paraffin. Two-micrometer sections were stained with Sirius red and Periodic acid-Schiff. The stained sections were examined by light microscopy (BZ-9000, Keyence, Osaka, Japan) in a blinded manner. Six sections were randomly selected, and the percentage of renal fibrosis was quantitated using the measurement module of BZ-X analyzer 1.3.1.1 (Hybrid Cell Count, Keyence). The total number of LVs was counted in all fields. LVs were identified as LV endothelial hyaluronan receptor-1 (LYVE-1)positive vessels labeled by immunohistochemistry. Capillaries were identified as positive for CD31 by immunohistochemistry. The vessel density in each area was expressed as the number or percentage area of vessels per $\mathrm{mm}^{2}$. Total macrophages were counted as F4/80-positive cells labeled by immunohistochemistry. Six sections were randomly selected, and the percentages of macrophage-positive areas were quantitated using the measurement module of BZ-X analyzer 1.3.1.1. M1 macrophages were identified as F4/80 and MCP-1 double-positive cells labeled by immunofluorescence staining. M2 macrophages were identified as F4/80 and ym-1 doublepositive cells labeled by immunofluorescence staining. Six sections were randomly selected, and the numbers of F4/80 and MCP-1 or ym-1 double-positive cells were calculated at $\times 400$ magnification.

\section{Immunohistochemistry}

Mouse renal tissues were prepared and stained as described previously. ${ }^{21}$ Primary antibodies were as follows: rabbit polyclonal anti-CD31 antibody (ab28364, 1:200, Abcam,

Figure 1 Administration of vascular endothelial growth factor (VEGF)-C in unilateral ureteral obstruction (UUO) mice. (a) Experimental protocol. Sevenweek-old male C57BL/6J mice were divided into the control group $(n=8)$, UUO+PBS group $(n=8)$, and UUO+VEGF-C group ( $n=8)$. VEGF-C was administered at $10 \mu \mathrm{g} / 100 \mathrm{~g}$ per day using osmotic pumps for 14 days. PBS was administered at the same volume applied to the UUO+VEGF-C group using the osmotic pump for 14 days. (b) Plasma levels of human VEGF-C after administration. Plasma levels of human VEGF-C were determined by an enzyme-linked immunosorbent assay (ELISA). One-way analysis of variance (ANOVA), **P<0.01 versus CTR and UUO+PBS groups. (c) VEGFR-3 was expressed specifically in lymphatic vessels (LVs). Representative images of LV endothelial hyaluronan receptor (LYVE)-1 and VEGFR-3, and merged images of the kidney in the UUO mouse obtained by confocal laser microscopy are shown. LYVE-1, green; VEGFR-3, red. LYVE-1-positive LVs were seen near blood vessels in the UUO kidney. Arrowheads, lymphatic endothelial cells (LECs); asterisks, nonspecific signals. 
a
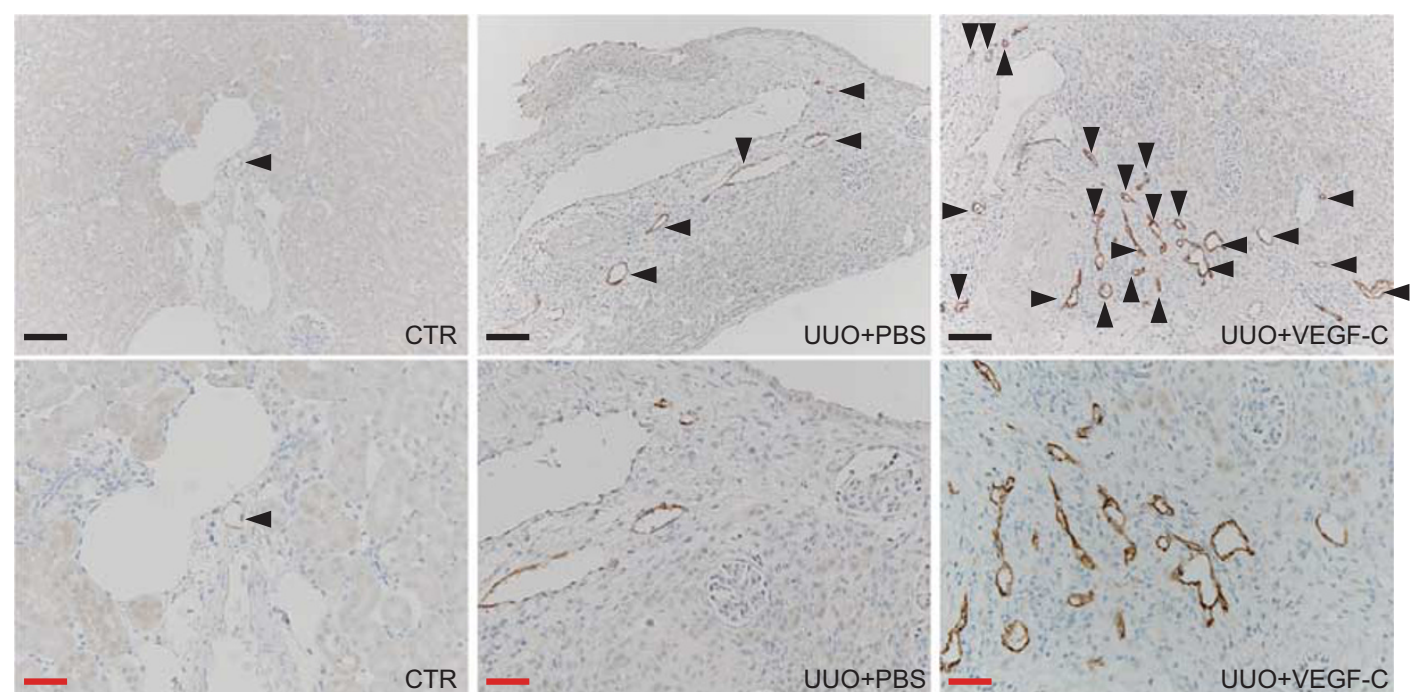

CTR
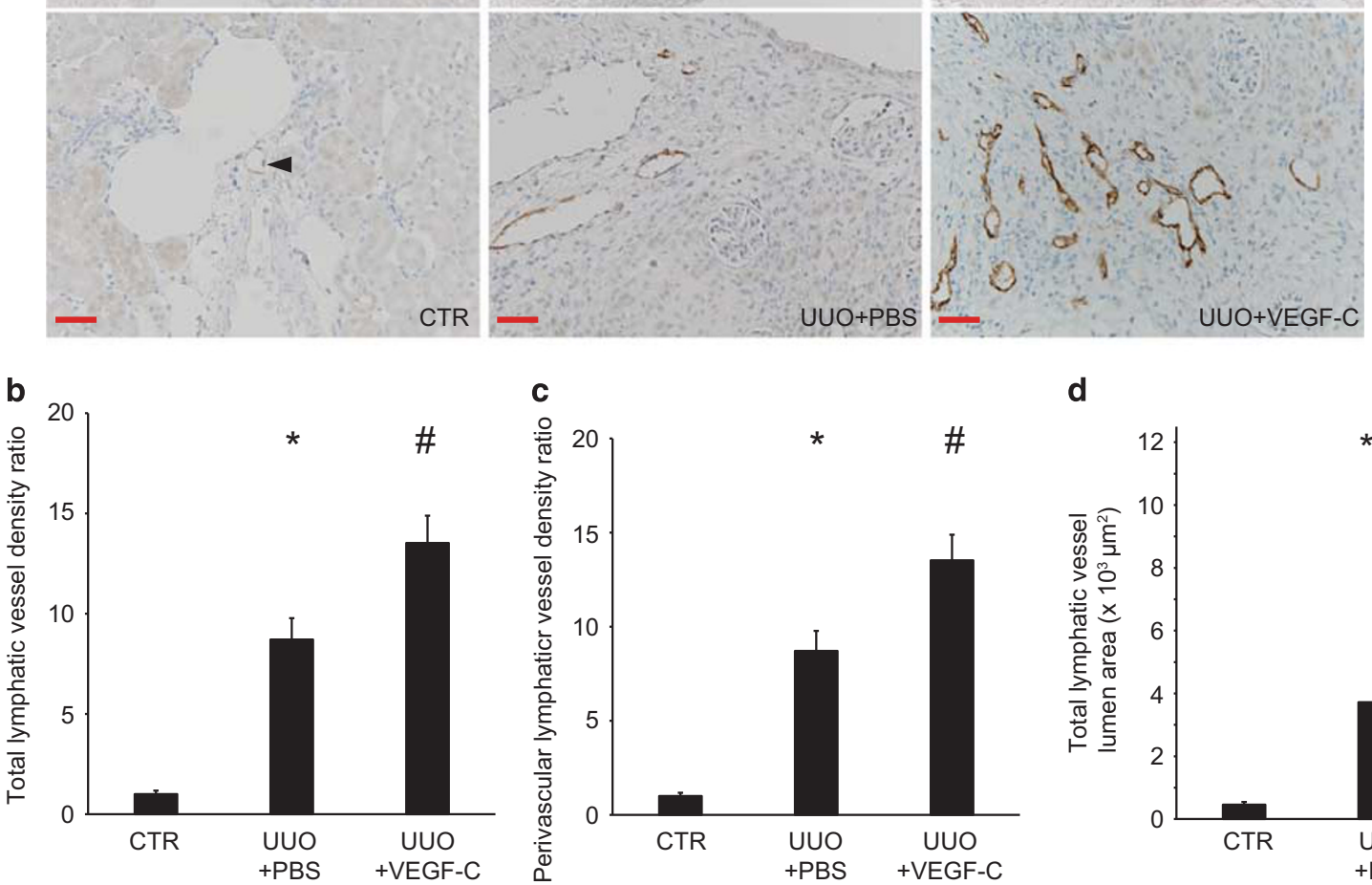

d

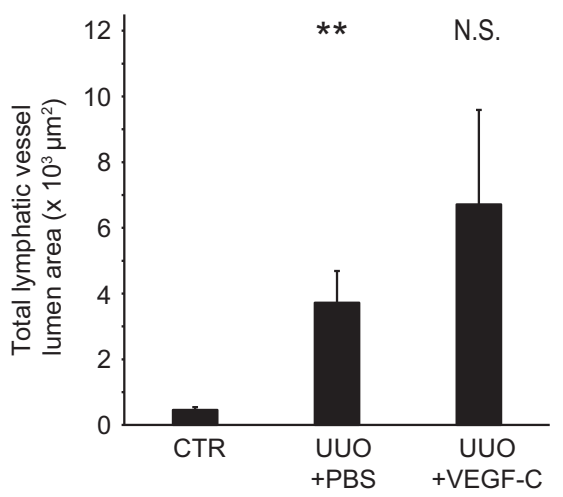

e

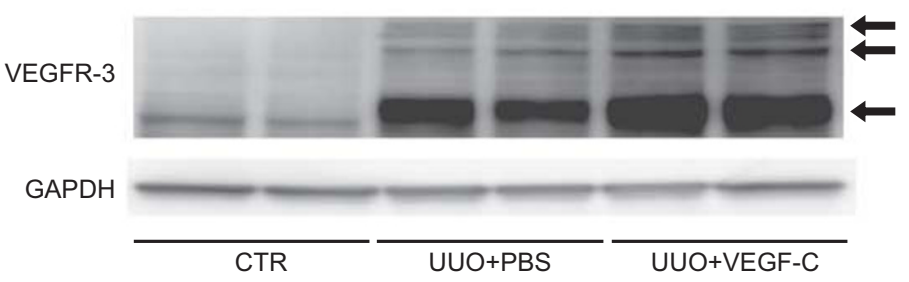

g
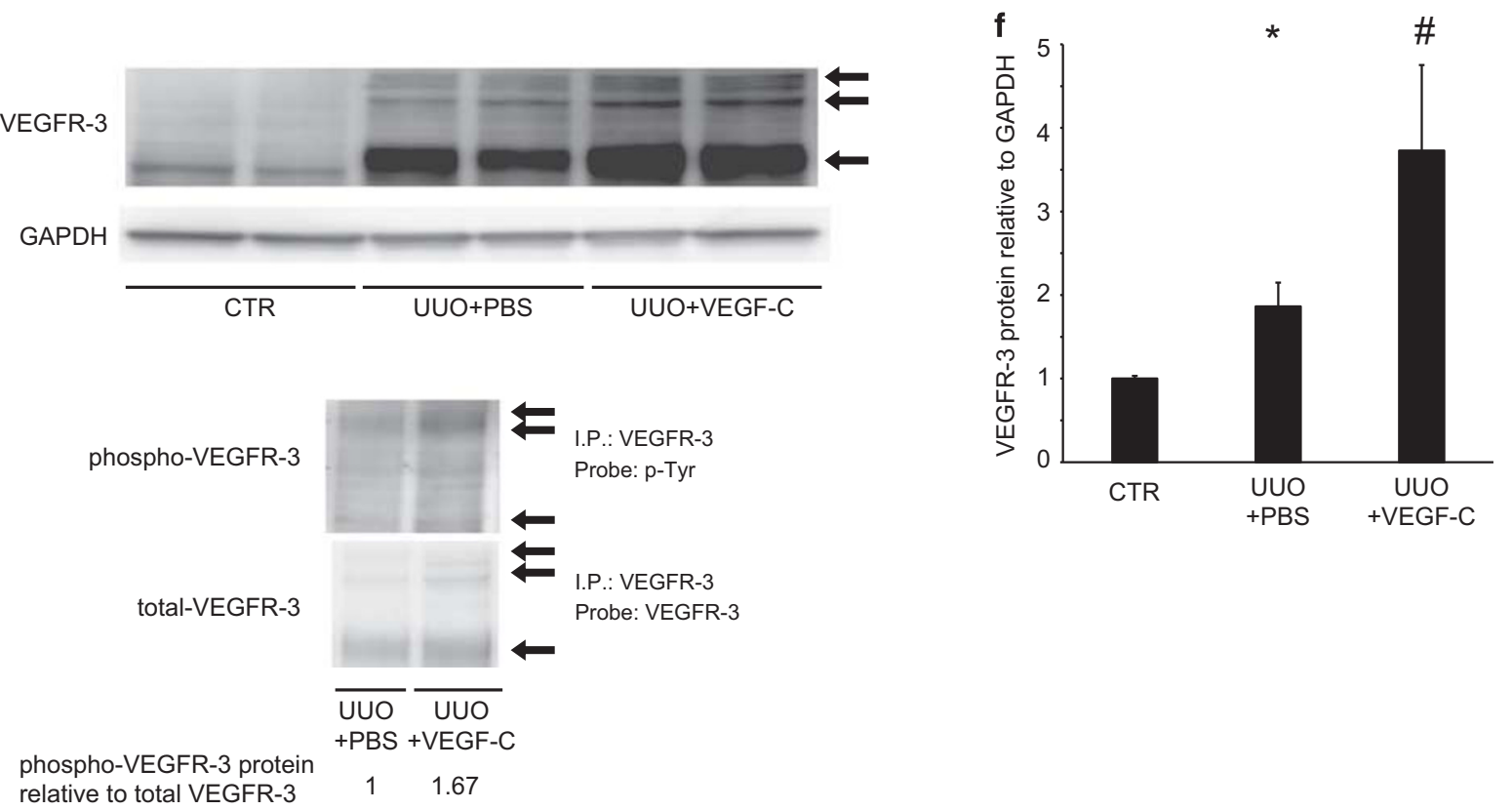
Cambridge, MA); rabbit polyclonal antibody against human LYVE-1 $\left(0.99 \mu \mathrm{g} / \mathrm{ml}\right.$, author produced $\left.{ }^{22}\right)$; goat monoclonal anti-mouse VEGFR-3 antibody (AF743, 1:15, R\&D Systems); rat monoclonal anti-F4/80 (MCA497G, 1:1000, Bio-Rad AbD Serotec, Oxford, UK); goat polyclonal anti-MCP-1 (sc-1785, 1:50, Santa Cruz Biotechnology, Dallas, TX, USA); rabbit polyclonal anti-Ym-1 (\#60130, 1:50, STEMCELL technologies, Vancouver, Canada). Secondary antibodies were as follows: peroxidase-conjugated anti-rabbit and goat (424132 and 424162, Histofine Simple Stain MAX PO; Nichirei, Tokyo, Japan); Alexa 488-conjugated chicken anti-rabbit IgG (A21441, 1:250, Life Technologies, Carlsbad, CA); Alexa 568-conjugated donkey anti-goat IgG (A11057, 1:250, Life Technologies); Alexa 488-conjugated goat anti-rabbit IgG (A11008, 1:250, Life Technologies); Alexa 568-conjugated goat anti-rat IgG (A11077, 1:250, Life Technologies); Alexa 488-conjugated donkey anti-goat IgG (A11055, 1:250, Life Technologies); Alexa-594 conjugated donkey anti-rat IgG (A21209, 1:250, Life Technologies). After counterstaining with 4',6-diamino-2-phenylindole, images were obtained by light microscopy (BX53; Olympus), the BZ-9000 digital microscope system (Keyence), or confocal laser scanning microscopy (LSM 780; Carl Zeiss, Oberkochen, Germany).

\section{VEGF-C Enzyme-linked Immunosorbent Assay (ELISA)}

Human VEGF-C concentrations in mouse plasma were determined using an ELISA (DVEC00, Quantikine Immunoassay systems; R\&D Systems). Mouse VEGF-C concentrations in mouse plasma were also determined using an ELISA (OKEH00240, Aviva Systems Biology, Corp. San Diego, CA, USA).

\section{Western Blotting and Immunoprecipitation}

Renal tissues in lysis buffer (RIPA buffer with a Protease inhibitor cocktail; 08714-04, Nacalai Tesque, Kyoto, Japan) containing a phosphatase inhibitor (06863-01, Nacalai Tesque) were homogenized twice for $3 \mathrm{~min}$ at $30 \mathrm{~Hz}$ using a TissueLyser (Qiagen, Hilden, Germany). The homogenate was centrifuged at $10000 \mathrm{~g}$ for $5 \mathrm{~min}$ at $4{ }^{\circ} \mathrm{C}$, and the collected supernatant was analyzed. Protein samples $(15 \mu \mathrm{g})$ were separated by SDS-polyacrylamide gel electrophoresis on 5-20\% polyacrylamide gradient gels (2331830, PAGEL; Atto, Tokyo, Japan) and blotted onto a polyvinylidene difluoride membrane using an iBlot system (Life Technologies). Primary and secondary antibodies were diluted in Can Get Signal Solution 1 and 2 (NKB-101, Toyobo, Osaka, Japan). After preincubation in blocking solution (03953-95, Blocking One, Nacalai Tesque) for $30 \mathrm{~min}$, the membranes were incubated overnight at $4{ }^{\circ} \mathrm{C}$ with the following primary antibodies: rabbit polyclonal anti-Collagen 1 (AB765P, 1:500, Millipore); goat polyclonal anti-VEGFR-3 (AF743, 1:1000, R\&D systems); rabbit polyclonal anti-transforming growth factor- $\beta 1$ (TGF- $\beta 1$ ) (18978-1-AP, 1:1000, Cell Signaling, Rosemont, IL, USA); rabbit monoclonal anti-phospho-VEGFR-2 (Tyr-1175; \#2478 S 1:1000, Cell Signaling Technology, Danvers, MA, USA); goat polyclonal anti-vascular cell adhesion molecule-1 (VCAM-1) (C-19) (sc-1504, 1:1000, Santa Cruz Biotechnology); mouse monoclonal anti-glyceraldehyde-3-phosphate dehydrogenase (GAPDH; ab8245, 1:5000, Clone 6C5; Abcam). Immunoreactive bands were detected using a chemiluminescence imaging system (AE-9300 Ez-capture MG, Atto). The density of each band was analyzed by ImageJ software (http://rsb.info.nih.gov/ij/).

One milligram of proteins from the kidneys of UUO mice administered the vehicle or VEGF-C was isolated using RIPA buffer and incubated with Protein A/G PLUS-Agarose Immunoprecipitation reagent (sc-2003, Santa Cruz Biotechnology) and $5 \mu \mathrm{g}$ anti-VEGFR-3 antibody (R\&D systems). Bound proteins were eluted, denatured, and separated on 5-20\% SDS-polyacrylamide gels. After electroblotting,

Figure 2 Vascular endothelial growth factor (VEGF)-C induces proliferation of lymphatic vessels (LVs) in unilateral ureteral obstruction (UUO) mice. (a) Representative images of LV endothelial hyaluronan receptor (LYVE)-1 immunostaining in the mouse kidney. Lymphangiogenesis was enhanced significantly in the UUO+PBS group compared with the control group. After VEGF-C administration, LVs proliferated in the UUO+VEGF-C group. Arrowheads, LVs. Black scale bar $=200 \mu \mathrm{m}$. Red scale bar $=80 \mu \mathrm{m}$ (b) Density of total LVs determined by LYVE-1 immunohistochemical staining. The vessel density in each area is expressed as the number of vessels per $\mathrm{mm}^{2}$. CTR, control $(n=8)$; UUO+PBS $(n=8)$; UUO+VEGF-C ( $\left.n=8\right)$. One-way analysis of variance (ANOVA), ${ }^{*} P<0.05$ versus CTR, ${ }^{\#} P<0.05$ versus CTR and UUO+PBS. (c) Density of perivascular LVs determined by LYVE- 1 immunohistochemical staining. The vessel density in each area is expressed as the number of vessels per $\mathrm{mm}^{2}$. CTR, control $(n=8) ; \mathrm{UUO}+\mathrm{PBS}(n=8)$; UUO+VEGF-C ( $n=8)$. One-way ANOVA, ${ }^{*} P<0.05$ versus CTR, ${ }^{\#} P<0.05$ versus CTR and UUO+PBS. (d) Total LV luminal area determined by LYVE- 1 immunohistochemical staining. CTR, control $(n=8)$; UUO+PBS $(n=8)$; UUO+VEGF-C $(n=8)$. One-way ANOVA, $* P<0.05$ versus CTR, N.S. not significant versus UUO+PBS. (e) Western blotting of VEGFR-3 protein in the kidney. GAPDH protein expression was examined as an internal control. VEGFR-3 stained as three bands, a $125 \mathrm{kDa}$ cleaved form and two uncleaved forms of 175 and $195 \mathrm{kDa}$ that differ in the degree of glycosylation. Black arrows indicate each band. ${ }^{44}$ ( $f$ ) Quantification of VEGFR-3 by western blotting $\left(n=8\right.$ each). One-way ANOVA. ${ }^{*} P<0.05$ versus $C$ TR, ${ }^{*} P<0.05$ versus $C$ TR and UUO+PBS. (g) Immunoprecipitation (IP) of phospho-VEGFR-3 and western blot analysis of VEGFR-3. VEGFR-3 phosphorylation levels were measured in the kidneys of UUO mice administered the vehicle or VEGF-C. (h) Immunostaining of CD31 in the mouse kidney. Capillaries in the kidney on day 14 were labeled with an anti-CD31 antibody. Representative images are shown. Arrows, capillaries. Scale bar $=100 \mu \mathrm{m}$. (i) Quantification of capillaries by CD31 immunostaining $(n=8)$. One-way ANOVA, ${ }^{*} P<0.05$ versus CTR. Not significant (NS) versus UUO+PBS. Scale bar $=50 \mu \mathrm{m}$. (j) Western blot analysis of phosphor-VEGFR-2 (pVEGFR-2). GAPDH was examined as an internal control. (k) Quantification of pVEGFR-2 protein expression. CTR ( $n=8$ ); UUO+PBS $(n=8) ;$ UUO+VEGF-C $(n=8)$. One-way ANOVA, ${ }^{*} P<0.05$ versus CTR. Not significant (NS) versus UUO+PBS. 
h
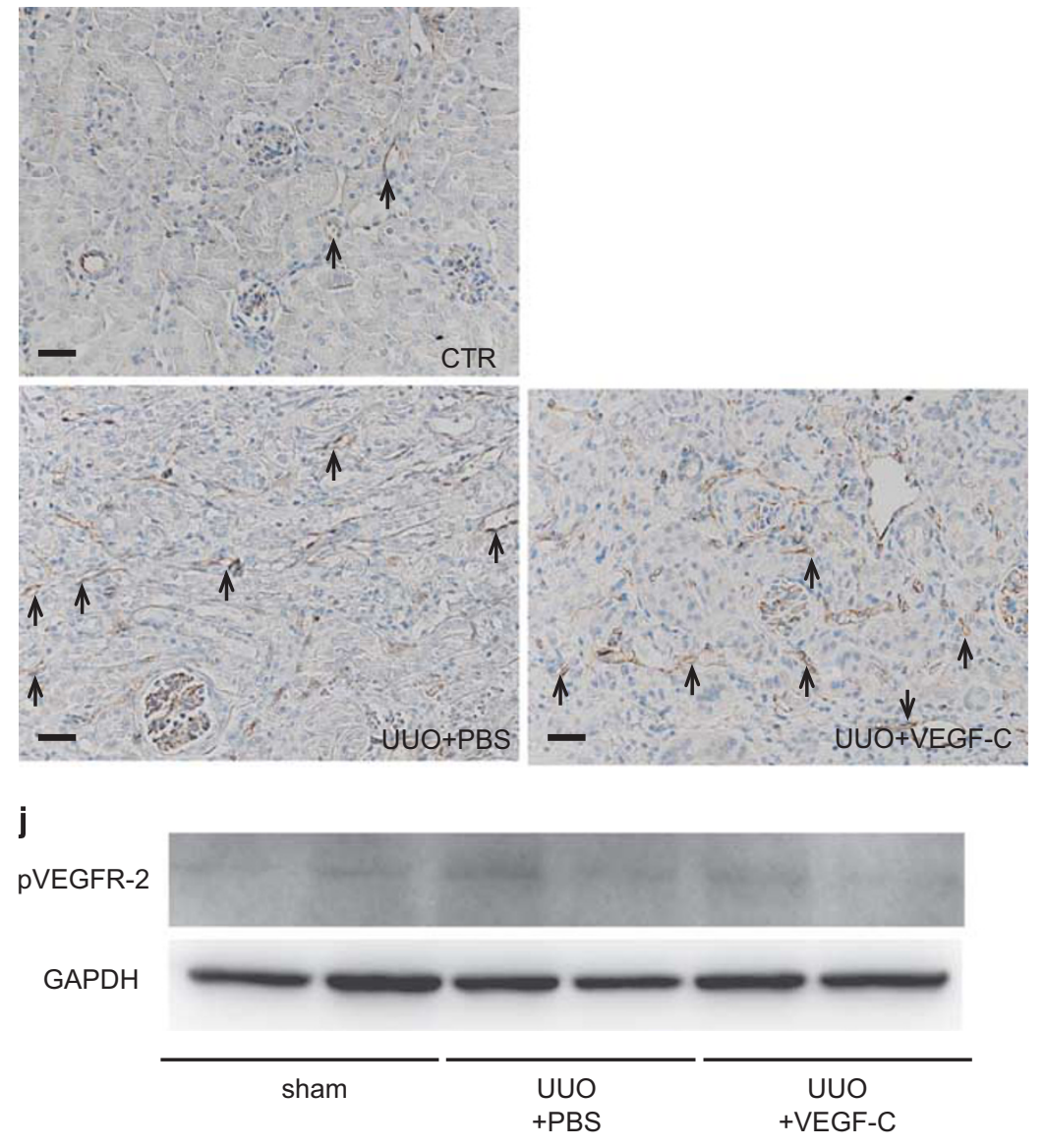

i
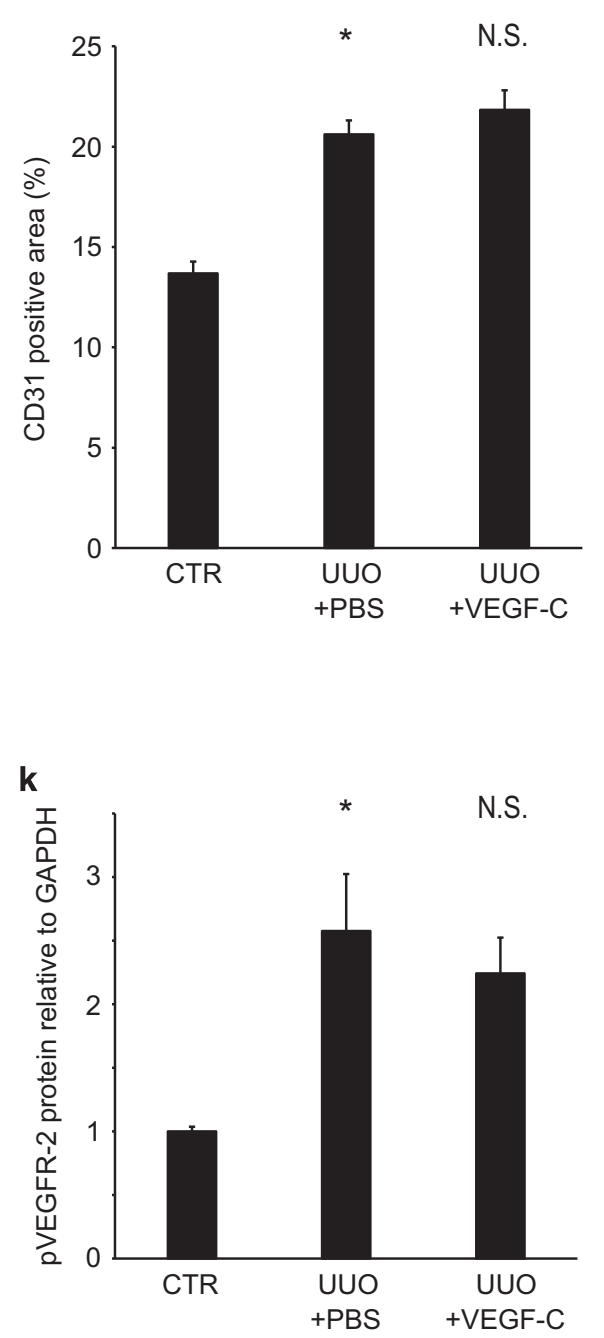

Figure 2 Continued.

proteins were detected using antibodies against phosphotyrosine (05-321, EMD Millipore, Billerica, MA, USA) or VEGFR-3.

\section{In Vitro Experiments}

Human lung lymphatic microvascular endothelial cells (HMVEC-LLys) were purchased from Lonza (Walkersville, $\mathrm{MD}, \mathrm{USA}$ ) and maintained in EGM-2MV medium consisting of EBM-2 basal medium, 5\% fetal bovine serum, and SingleQuots containing growth factors (VEGF, fibroblast growth factor-2, insulin-like growth factor-I, and epidermal growth factor). All experiments were performed between passages 4 and 10. Under subconfluent conditions, cells were incubated with $100 \mathrm{ng} / \mathrm{ml}$ recombinant human VEGF-C (R\&D Systems) diluted in PBS. For RT-PCR, cells were collected at $24 \mathrm{~h}$ after incubation. For western blotting, cells were collected at $48 \mathrm{~h}$ after incubation.

\section{Cell Viability Assay}

To assess HMVEC-LLys viability, HMVEC-LLys in EGM-2 were seeded into 96 -well plates and incubated at $37^{\circ} \mathrm{C}$ with $5 \% \mathrm{CO}_{2}$. After 1 day, the medium was changed to complete EGM-2MV with or without $100 \mathrm{ng} / \mathrm{ml}$ VEGF-C. After 4 days, HMVEC-LLys viability was assessed using a WST-8 [2-(2methoxy-4-nitrophenyl)-3-(4-nitrophenyl)-5-(2,4-disulfophenyl)-2H-tetrazolium, monosodium salt] assay (\#07553-15, Nacalai Tesque).

\section{In Vitro Wound Healing Assay}

HMVEC-LLys were cultured in EGM-2MV medium to confluency. Then, the monolayers were wounded (T0) in a line across the well with a $200-\mu l$ pipette tip. Medium was replaced with or without $100 \mathrm{ng} / \mathrm{ml}$ VEGF-C in complete EGM-2MV. The scratches were photographed at T0 and after $12 \mathrm{~h}$ (T12) using an inverted microscope (ECLIPSE Ti-U; 
a

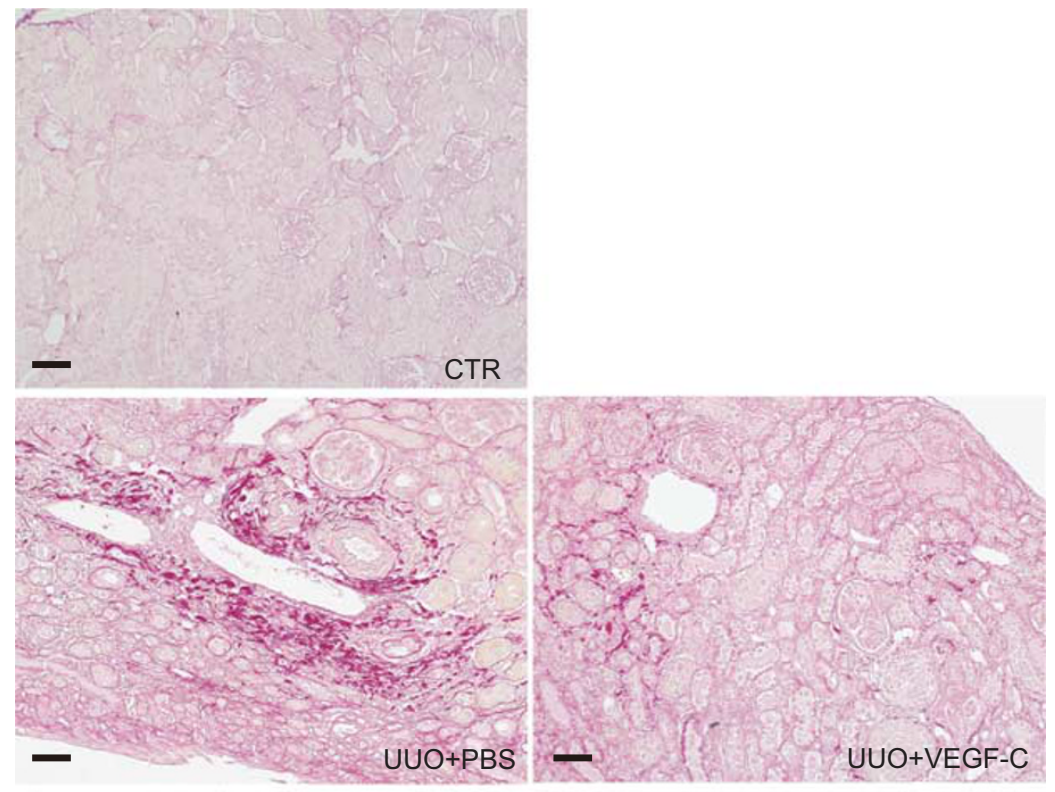

C

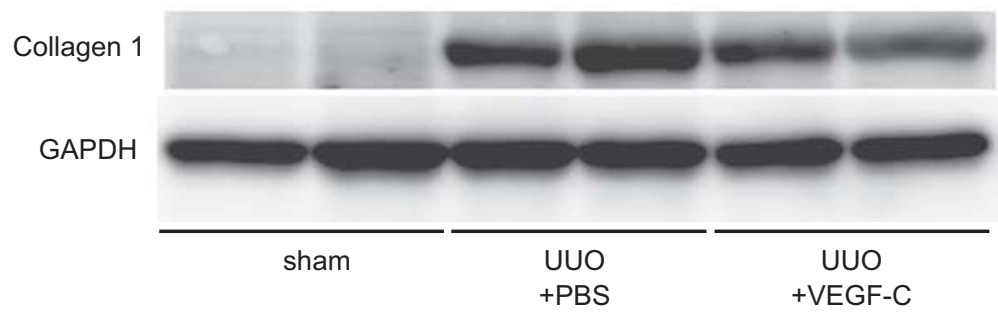

b
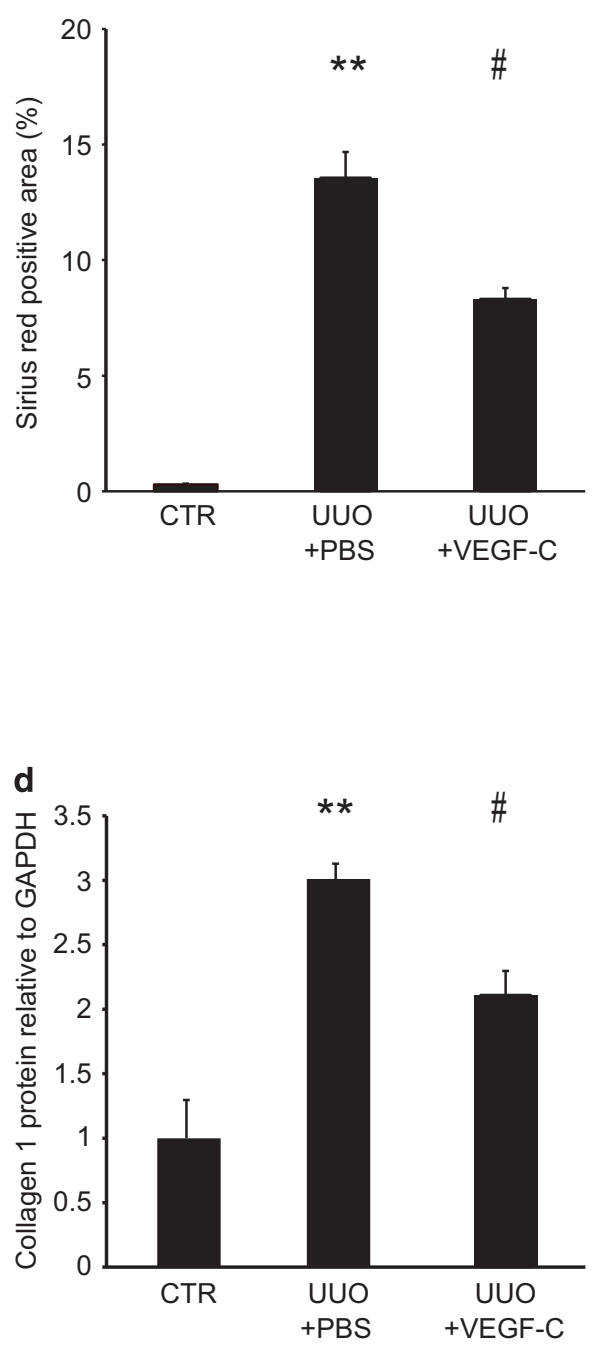

Figure 3 Interstitial fibrosis of mouse kidney is attenuated by vascular endothelial growth factor (VEGF)-C administration. (a) Sirius red staining of the mouse kidney. Representative images are shown. Scale bar $=50 \mu \mathrm{m}$. (b) Quantification of the fibrotic area determined by Sirius red staining. CTR, control $(n=8)$; UUO+PBS $(n=8)$; UUO+VEGF-C $(n=8)$. One-way analysis of variance (ANOVA), ${ }^{* *} P<0.01$ versus CTR. ${ }^{*} P<0.05$ versus UUO+PBS. (c) Western blot analysis of collagen 1. GAPDH was examined as an internal control. (d) Quantification of collagen 1 protein expression. UUO $(n=8)$; UUO+VEGF-C $(n=8)$. One-way ANOVA, ${ }^{* *} P<0.01$ versus CTR. ${ }^{*} P<0.05$ versus UUO+PBS.

Nikon, Tokyo, Japan) and NIS-Elements software (Nikon; $\times 40$ magnification). Two images were obtained per well, and at least duplicate wells per condition were analyzed using ImageJ software. The wounded area per field was individually assessed and averaged per well, and the percentage area covered was calculated for each well on an individual basis. Experiments were performed three different times with individual analyses.

\section{Real-Time PCR}

Total RNA was extracted from the kidney lysate or cells, and cDNA was synthesized. Real-time PCR was performed using SYBR Premix Ex Taq (RR420A, Takara Bio Inc., Otsu, Japan) and a 7500 Real Time PCR System (Applied Biosystems, Foster, CA, USA). Relative expression levels of procollagen-1, alpha smooth muscle actin, tumor necrosis factor (TNF)alpha, and monocyte chemoattractant protein-1 (MCP-1) in the kidney lysate and those of intercellular adhesion molecule-1 (ICAM-1), VCAM-1, and E-selectin in cells were determined by the $\triangle \Delta C T$ method using GAPDH as an internal reference.

\section{Statistical Analysis}

Statistical analysis was performed using SPSS version 19.0 software (IBM, Armonk, NY, USA). Results are presented as the mean \pm s.e.m. Differences between two groups were 
compared by the Student's $t$-test. Differences among groups were compared by one-way analysis of variance (ANOVA), followed by Dunnett's test. Dose-dependent differences were compared by one-way ANOVA, followed by Tukey's honestly significant difference tests. For all statistical tests, a two-tailed $P$-value of $<0.05$ was considered as statistically significant. a

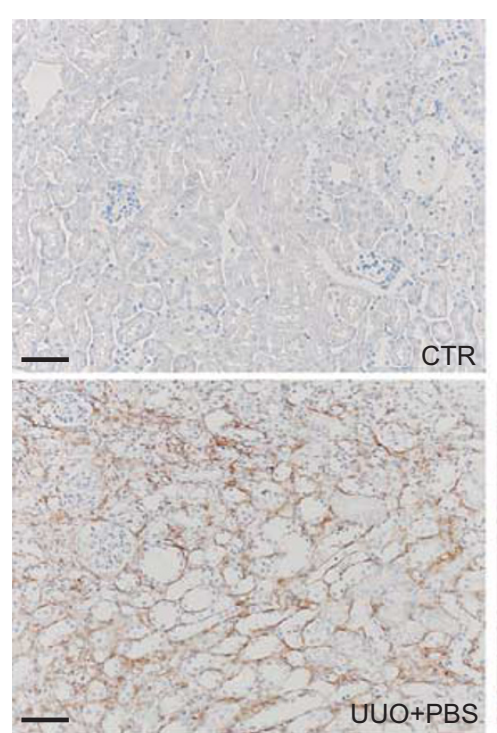

C

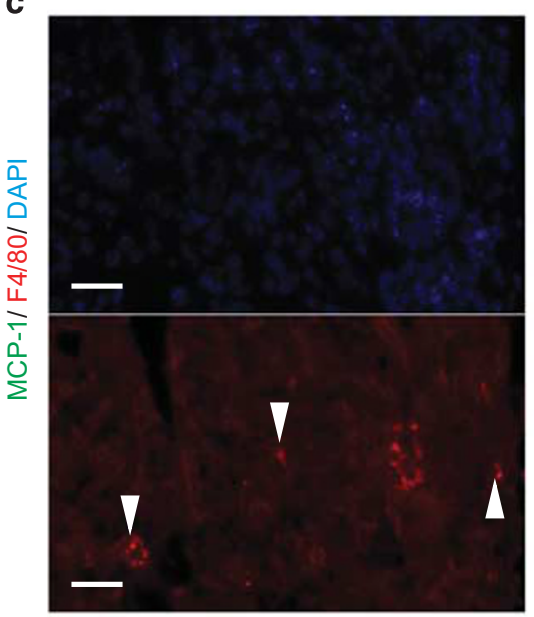

e

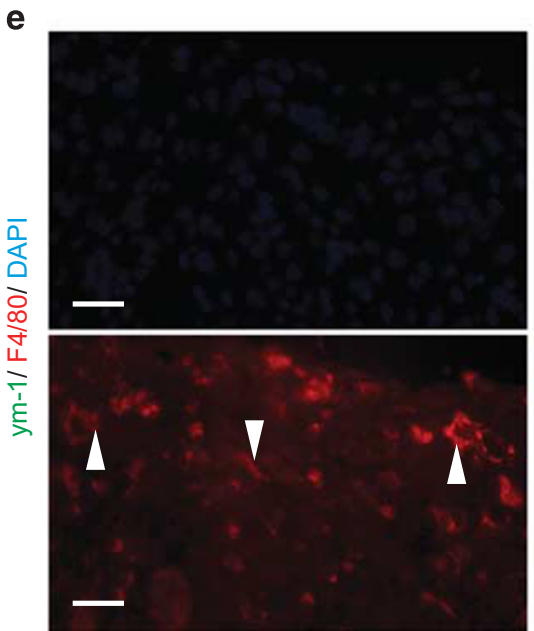

b

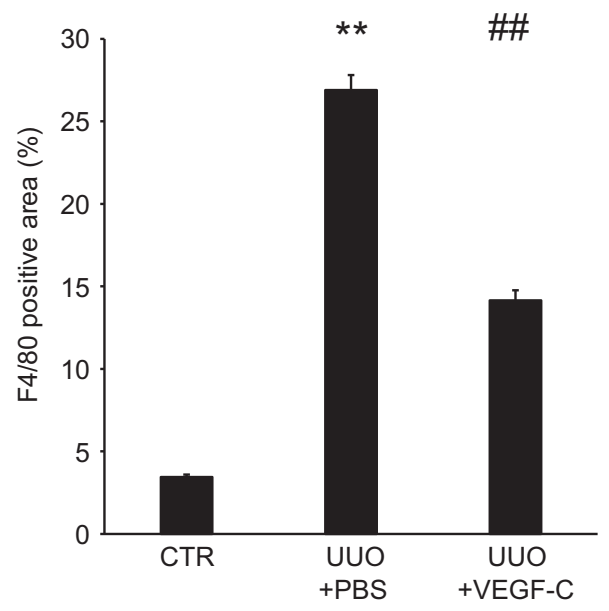

d

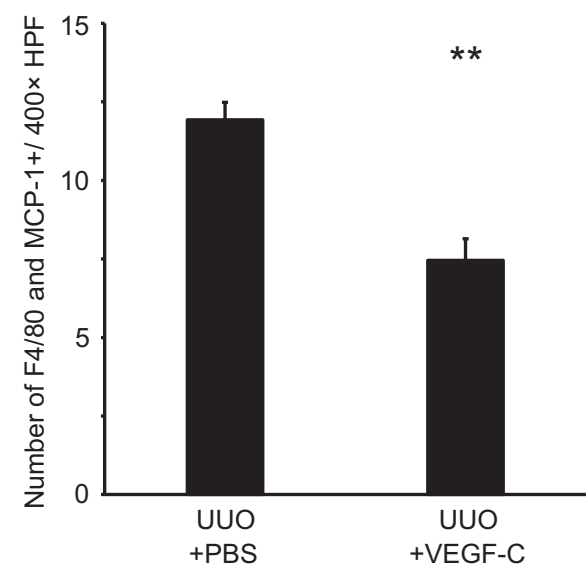

f

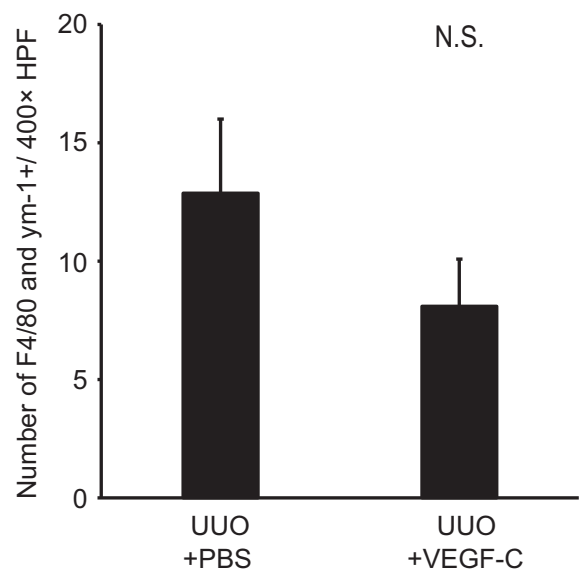




\section{RESULTS}

Systemic Administration of Recombinant Human VEGF-C in Mice

First, we confirmed that recombinant human VEGF-C was effectively delivered into murine blood. An approximate threefold increase of VEGF-C levels in plasma was observed in the UUO+VEGF-C group (Figure 1b) using the human VEGF-C ELISA kit. VEGF-C levels of the UUO+VEGF-C group were significantly elevated by continuous administration of recombinant human VEGF-C. Despite not administrating human VEGF-C in CTR and UUO+PBS groups, human VEGF-C levels in mouse plasma were around $200 \mathrm{pg} /$ $\mathrm{ml}$. Because even $1000 \mathrm{pg} / \mathrm{ml}$ recombinant mouse VEGF-C was less than the minimum detectable limit of the ELISA kit, these human VEGF-C concentrations in CTR and UUO+PBS groups were considered to be due to nonspecific proteins in mouse plasma. Furthermore, to investigate the possibility of endogenous mouse VEGF-C secretion, we measured mouse VEGF-C levels in plasma using the mouse VEGF-C ELISA. In all groups, mouse endogenous VEGF-C levels were almost all below the detection limit or $<50 \mathrm{pg} / \mathrm{ml}$, and there was no difference among groups (Supplementary Figure 1). We concluded that mouse endogenous VEGF-C secretion was insignificant in the UUO model mice, and administration of human VEGF-C increased plasma VEGF-C levels in mice.

\section{VEGFR-3 is Expressed in LECs, but not in Vascular Endothelial Cells}

Consistent with a previous report, ${ }^{23}$ VEGFR-3 was expressed predominantly in LECs, but not in vascular endothelial cells (Figure 1c). VEGFR-3 was expressed in both large and small LYVE-1-positive LVs.

\section{VEGF-C Administration Enhances Proliferation of LVs in the UUO Kidney}

Next, we examined the relationship between renal fibrosis and lymphangiogenesis. The number of LVs was determined by LYVE-1 immunohistochemistry. As reported previously, ${ }^{24}$ lymphangiogenesis was enhanced significantly in the UUO + PBS group compared with the control group (Figures $2 \mathrm{a}$ and b). Proliferation of LVs in the UUO+VEGF-C group was significantly higher than that in the UUO+PBS group (Figures $2 \mathrm{a}$ and $\mathrm{b}$ ). Total and perivascular lymphatic densities were increased by VEGF-C administration. Therefore, newly formed perivascular LVs extended from existing LVs because of the VEGF-C-VEGF-R3 signaling pathway activated by
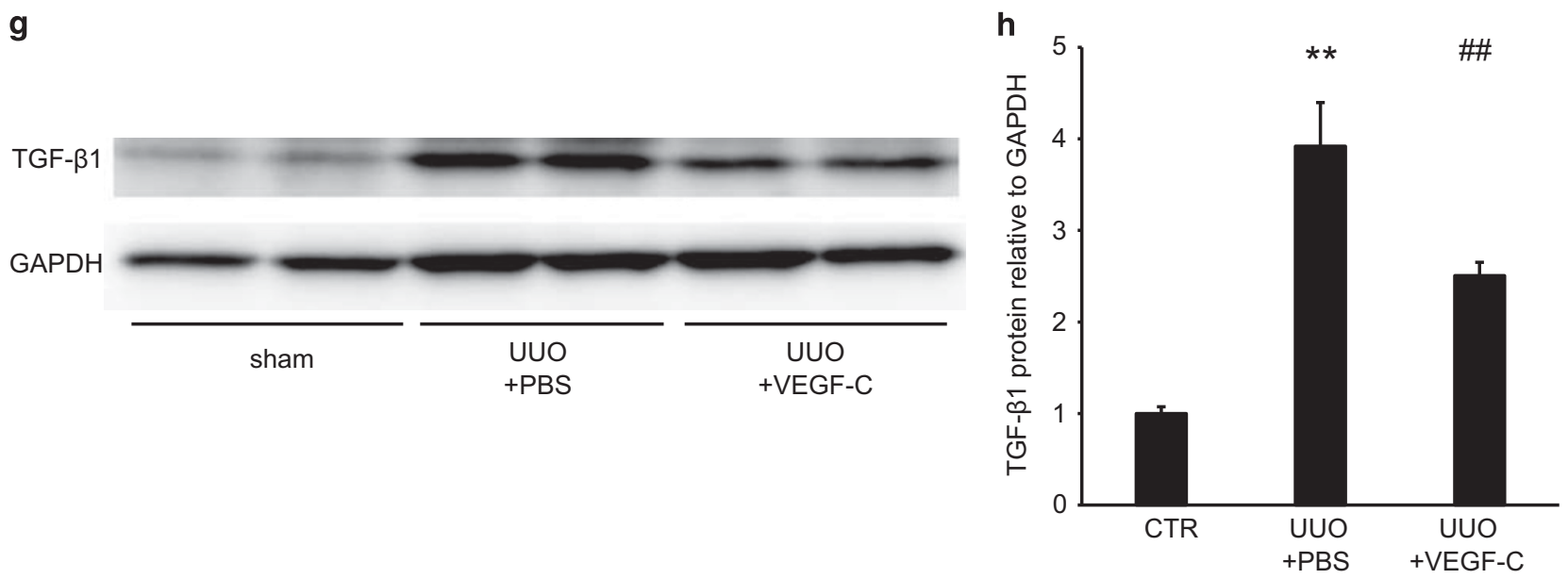

Figure 4 Continued.

Figure 4 Vascular endothelial growth factor (VEGF)-C administration reduces inflammation of the kidney. (a) F4/80 immunostaining of the mouse kidney. Representative images of F4/80 staining in unilateral ureteral obstruction (UUO)+PBS and UUO+VEGF-C groups. Scale bar $=100 \mu \mathrm{m}$. (b) Quantification of the F4/80-positive area. The percentage of the F4/80-positive area is shown. CTR, control $(n=8)$; UUO $(n=8)$; UUO+VEGF-C $(n=8)$. Oneway analysis of variance (ANOVA), ${ }^{* *} P<0.01$ versus CTR. ${ }^{\# \#} P<0.01$ versus UUO+PBS. (c) Double immunofluorescence staining of macrophages for F4/80 and monocyte chemotactic protein-1 (MCP-1). Immunofluorescence images show staining for nuclei (4',6-diamino-2-phenylindole (DAPI); blue), F4/80 (red), and MCP-1 (green). Arrowheads indicate double-positive macrophages expressing F4/80 and MCP-1. (d) Number of F4/80 and MCP-1 doublepositive cells counted at $\times 400$ magnification. Scale bar $=20 \mu \mathrm{m}$. Student's $t$-test, ${ }^{* * P}<0.01$ versus UUO+PBS. (e) Double immunofluorescence staining of macrophages for F4/80 and ym-1. Immunofluorescence images show staining for nuclei (DAPl; blue), F4/80 (red), and ym-1 (green). Arrowheads indicate double-positive macrophages expressing F4/80 and ym-1. (f) Number of F4/80 and ym-1 double-positive cells counted at $\times 400$ magnification. Scale bar $=20 \mu \mathrm{m}$. Student's $t$-test, Not significant (NS) versus UUO+PBS. (g) Western blotting of transforming growth factor (TGF)- $\beta 1$ in UUO+PBS and UUO + VEGF-C groups at day 14. GAPDH was examined as an internal control. (h) Quantification of TGF- $\beta 1$ protein expression. UUO+PBS ( $n=8$ ); UUO+ VEGF-C $(n=8)$. One-way ANOVA, ${ }^{* *} P<0.01$ versus CTR. ${ }^{\# \# P}<0.01$ versus UUO+PBS. A full color version of this figure is available at the Laboratory Investigation journal online. 
VEGF-C administration. Furthermore, perivascular lymphangiogenesis was associated with the reduction of interstitial fibrosis and inflammation (Figures $2 \mathrm{~b}$ and $\mathrm{c}$ ). Although the total lymphatic luminal area in the UUO+VEGF-C group tended to increase to almost 1.8 times that in the vehicle group, it was not significant (Figure 2d). These results indicate that the exogenous VEGF-C did not affect LV dilatation. Consistent with LYVE-1 immunostaining, the expression of VEGFR-3, which is specific to LECs, was significantly increased in the UUO+VEGF-C group compared with the UUO group (Figures 2e and f). Furthermore, after VEGF-C administration, VEGFR-3 phosphorylation measured by immunoprecipitation was increased in the UUO +VEGF-C group (Figure 2g). However, VEGF-C did not enhance angiogenesis in the UUO+VEGF-C group. The density of capillaries was significantly increased in the UUO + PBS group compared with the control. After UUO surgery, because the kidney parenchyma becomes thin, the crosssectional area of the kidney shrinks markedly. In addition, the number of capillaries increases because of the inflammatory response due to pronounced acute interstitial nephritis induced by UUO surgery. There was no increase of capillaries in the UUO+VEGF-C group compared with the UUO+PBS group, although LVs had proliferated (Figures $2 \mathrm{~h}$ and i). After VEGF-C administration, VEGFR-2 phosphorylation was not increased in the UUO+VEGF-C group based on western blotting. (Figures $2 \mathrm{j}$ and $\mathrm{k}$ ) This result suggested that VEGF-C predominantly stimulated proliferation of LECs, but not vascular endothelial cells, in UUO kidneys.

\section{VEGF-C Suppresses Interstitial Renal Fibrosis in UUO Mice}

We explored the effect of VEGF-C-induced lymphangiogenesis in the UUO+VEGF-C group. Interstitial renal fibrosis determined by Sirius red staining was enhanced by 15 -fold in the UUO group compared with the control group, which was significantly attenuated by VEGF-C (Figures $3 \mathrm{a}$ and $\mathrm{b}$ ). The collagen 1 level determined by western blotting was significantly decreased by $30 \%$ in the UUO+VEGF-C group compared with the UUO group (Figures $3 \mathrm{c}$ and $\mathrm{d}$ ).

\section{VEGF-C Attenuates Interstitial Infiltration of Inflammatory Cells and Cytokine Production in the UUO Kidney}

We next focused on renal inflammation accompanied by fibrosis. Macrophages were labeled with the anti-F4/80 antibody in the kidneys (Figure 4a). The F4/80-positive area was significantly decreased by $48 \%$ in the UUO+VEGF-C group compared with the UUO+PBS group (Figures 4a and b). M1 macrophages identified by double immunofluorescence staining of F4/80 and MCP- 1 were decreased by $38 \%$ in the UUO+VEGF-C group compared with the UUO+PBS group (Figures $4 \mathrm{c}$ and $\mathrm{d}$ ). M2 macrophages identified by double immunofluorescence staining of $\mathrm{F} 4 / 80$ and $\mathrm{Ym}-1$ tended to decrease in the UUO+VEGF-C group compared with the UUO+PBS group, but there was no significance (Figures $4 \mathrm{e}$ and $\mathrm{f}$ ). At present, the effect of VEGF-C on macrophage polarization remains unclear. Consistent with the number of macrophages, a representative inflammatory cytokine in renal fibrosis, TGF- $\beta 1$, was significantly reduced in VEGF-C-treated UUO kidneys (Figures $4 \mathrm{~g}$ and $\mathrm{h}$ ).

\section{VEGF-C Stimulates Cell Proliferation and Expression of Adhesion Molecules in LECs}

To better understand how VEGF-C suppresses renal fibrosis via LECs, we examined the effect of VEGF-C on human primary LECs in vitro. The number of VEGF-C-treated LECs was increased by 1.2 -fold (Figure $5 \mathrm{a}$ ), which was consistent with the enhanced lymphangiogenesis in UUO mice treated with VEGF-C. To explore the migratory activity of LECs, we performed a wound healing assay. As a result, VEGF-C stimulated the migration of lymphatic cells (Figures $5 b$ and c). This finding suggests that LECs proliferate in response to VEGF-C and migrate to sites of inflammation in the kidneys. To establish whether lymphatic cell functions also change in response to VEGF-C, we examined gene expression of adhesion molecules ICAM-1, VCAM-1, and E-selectin. Expression of all three genes was significantly increased by 2-4.5-fold in response to VEGF-C (Figure 5d). VCAM-1 protein expression was also increased by 10 and $100 \mathrm{ng} / \mathrm{ml}$ VEGF-C treatment (Figures 5e and f). This result implies that VEGF-C enhances the expression of adhesion molecules that play a role in lymphocyte homing to LVs.

\section{DISCUSSION}

This study demonstrated that VEGF-C induced expression of VEGFR-3 and lymphangiogenesis in the interstitium and suppressed interstitial fibrosis in UUO kidneys. VEGF-C also reduced collagen 1 and TGF- $\beta 1$ expression as well as macrophage accumulation in the interstitium. In vitro, VEGF-C promoted LEC proliferation and migration, and induced expression of adhesion molecules ICAM-1, VCAM-1, and E-selectin in LECs. This study is the first to show that VEGF-C ameliorates interstitial fibrosis, and that LVs play an important role in draining inflammatory cells in the renal interstitium (Figure 6).

Lymphangiogenesis occurs at sites of tissue inflammation such as psoriasis, ${ }^{25}$ chronic airway inflammation, ${ }^{26}$ Crohn's disease, ${ }^{27}$ inflammatory bowel disease, ${ }^{28}$ and rheumatoid arthritis. ${ }^{29,30}$ In most organs, one of the main functions of LVs is to return fluid and macromolecules to the vascular system. ${ }^{6,23}$ Inflammation-induced lymphangiogenesis regulates fluid drainage, immune cell migration, and removal of inflammatory mediators, thereby ultimately accelerating the resolution of inflammation. ${ }^{11}$ We considered that lymphangiogenesis may have beneficial or adverse effects in various inflammatory environments. Previous studies have shown that a lack of lymphangiogenesis prolongs chronic inflammation in ischemic limbs. ${ }^{15}$ Lymphangiogenesis induced by VEGF-C ameliorates inflammatory diseases such as diabetic 
a

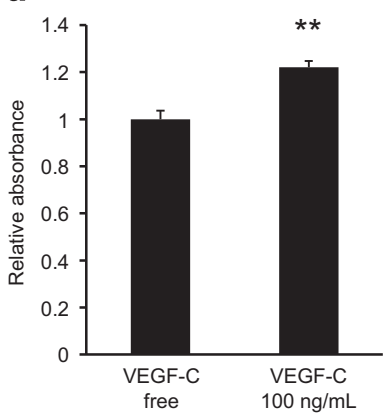

b

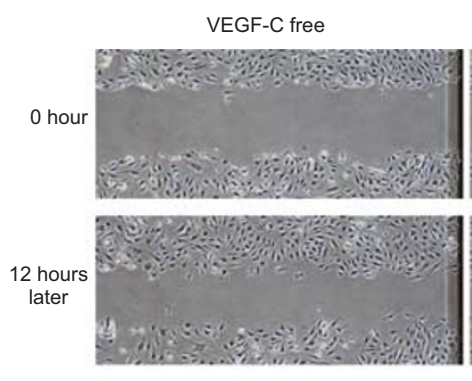

C

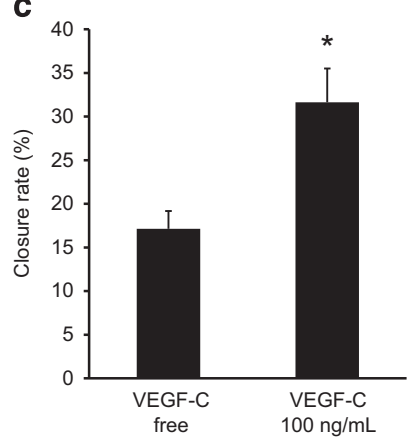

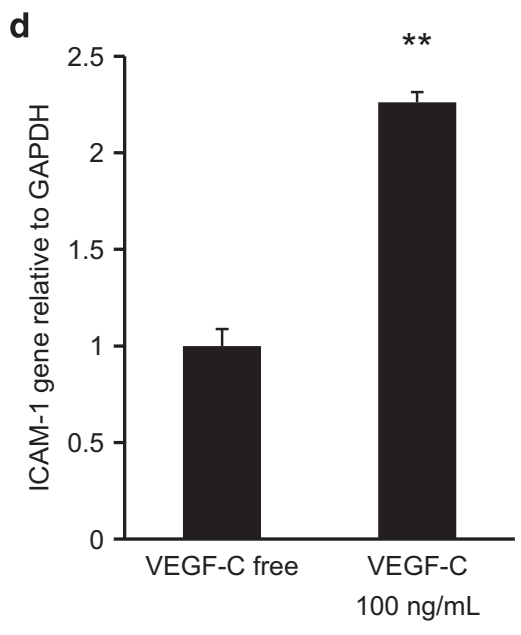

e

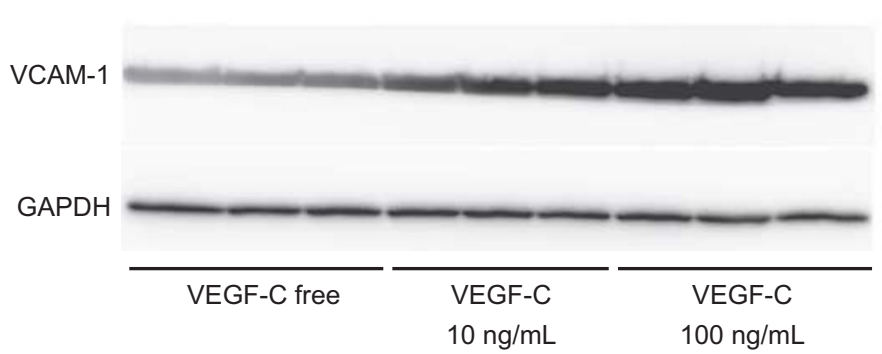

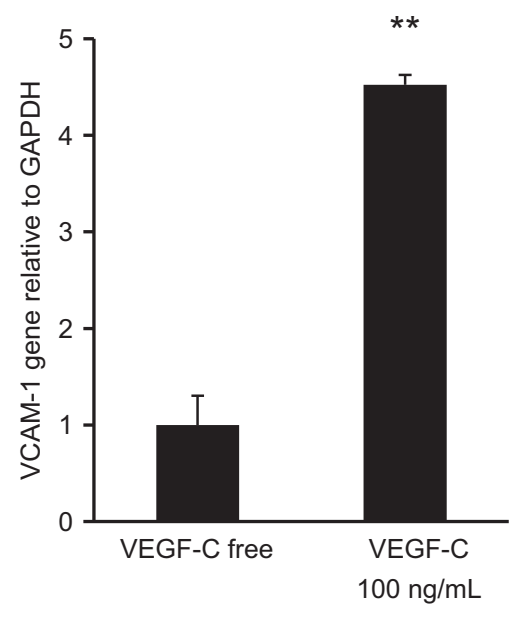

f

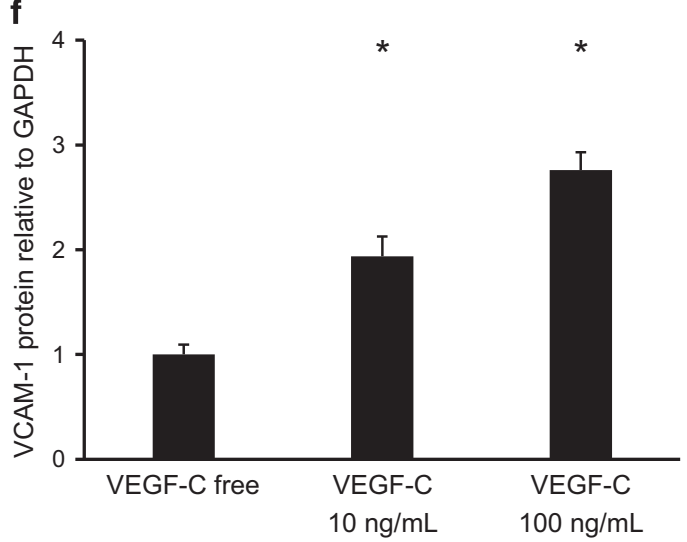

Figure 5 Vascular endothelial growth factor (VEGF)-C enhances cell proliferation, migration, and expression of adhesion molecules in lymphatic endothelial cells (LECs). (a) Human lung lymphatic microvascular endothelial cells (HMVEC-LLys) were seeded on 96 -well plates $\left(9 \times 10^{3}\right.$ cells/well) and treated with $100 \mathrm{ng} / \mathrm{ml}$ VEGF-C. After 4 days, cell viability was determined by a WST-8 assay $(n=3) .{ }^{*} P<0.01$. (b) HMVEC-LLys were seeded on six-well plates and grown to semi-confluence in complete medium, scratch-wounded, and allowed to recover with or without $100 \mathrm{ng} / \mathrm{ml} \mathrm{VEGF-C.} \mathrm{Images} \mathrm{shown}$ were obtained at $0 \mathrm{~h}$ (immediately after wounding) and at $12 \mathrm{~h}$ after wounding. (c) Quantification of the percentage of the wounded area that was closed at $12 \mathrm{~h}$ after wounding is shown as a representative experiment from at least three independent experiments. Student's $t$-test, ${ }^{*} P<0.05$. (d) Adhesion molecule gene expression associated with homing of inflammatory cells. Intercellular adhesion molecule-1, vascular cell adhesion molecule (VCAM)-1, and E-selectin mRNA expression levels were analyzed by real-time PCR. Values are the mean \pm s.e.m. Student's $t$-test, ${ }^{* *} P<0.01$ versus complete medium. (e) Western blotting of VCAM-1 in HMVEC-LLys treated with 0, 10, and $100 \mathrm{ng} / \mathrm{ml}$ VEGF-C. GAPDH was examined as an internal control. (f) Quantification of VCAM-1 protein expression. One-way analysis of variance (ANOVA), ${ }^{*} P<0.05 . n=3$ in each condition.

wounds ${ }^{31}$ and inflammatory bowel disease. ${ }^{3}$ In models of skin inflammation, lymphangiogenesis reduces skin edema. ${ }^{2}$ However, in the case of rejection in organ transplantation, lymphangiogenesis is involved in the maintenance of potentially detrimental alloreactive immune responses, because dendritic cells and antigen-presenting cells recovered from the proliferated LVs activate lymphocytes in lymphatic tissues and are mobilized locally through blood vessels. ${ }^{32-35}$ 


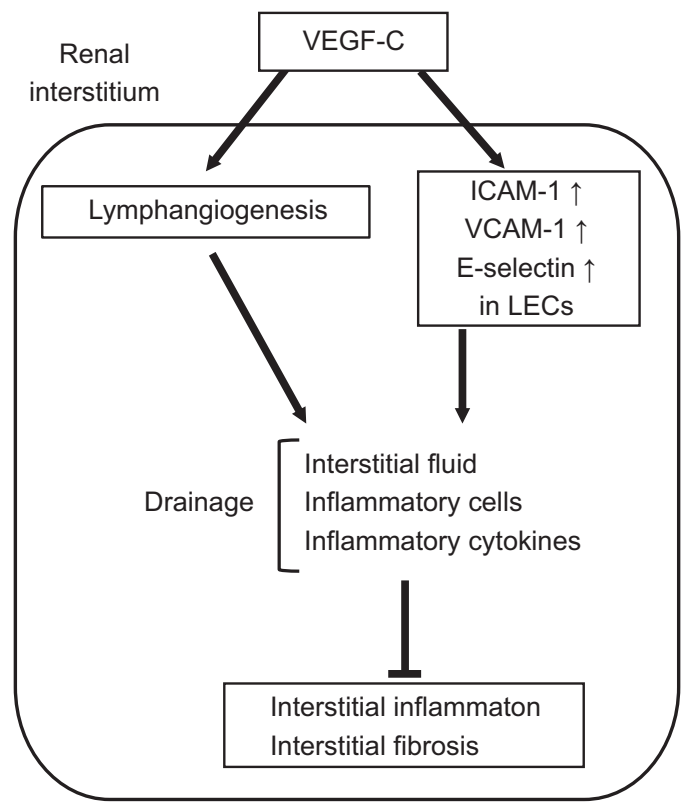

Figure 6 Possible mechanisms of vascular endothelial growth factor (VEGF)-C in the unilateral ureteral obstruction (UUO) kidney. VEGF-C is secreted by proximal tubule cells, collecting duct cells, and inflammatory cells during tubulointerstitial fibrosis of the UUO kidney. VEGF-C administration enhances lymphangiogenesis and lymphatic functions via VEGF-C-VEGFR-3 (VEGF receptor-3) signaling. Excess interstitial fluid, inflammatory cells, and inflammatory cytokines might drain from the renal interstitium through the developed lymphatic system. Therefore, interstitial inflammation is suppressed and subsequent interstitial fibrosis improves.

We considered that the difference in the inflammatory environment of each experimental model determines whether lymphangiogenesis has beneficial or adverse effects.

The UUO model used in this study is a model of severe interstitial nephritis. There is severe inflammation in the UUO kidney, followed by a marked rise in renal interstitial pressure. Lee et al. ${ }^{36}$ revealed that lymphangiogenesis correlates with the expression of VEGF-C and VEGF-D as well as renal fibrosis in UUO mice, and the sources of VEGF$\mathrm{C}$ are macrophages and tubular cells. However, the role of renal LVs in the progression of renal fibrosis is still unclear. They also showed that VEGF-C expression is higher in M2polarized macrophages than in M1-polarized macrophages. This finding suggests that LVs may be associated with fibrogenic processes. We revealed that lymphangiogenesis induced by VEGF-C suppressed macrophage infiltration, especially M1-polarized proinflammatory macrophages. The appropriate drainage effect of LVs may reduce inflammatory cells, pr-inflammatory cytokines, and excess fluid, and lead to suppression of renal fibrosis. However, insufficient drainage may lead to sustained renal inflammation and cause fibrosis. We revealed the LVs play a beneficial role in prevention of renal fibrosis. However, further study is needed to maintain the proper functions of LVs. We speculate that interstitial inflammatory cytokines, inflammatory cells, and interstitial fluid might be drained through the increased number of LVs by VEGF-C administration. The mRNA levels of procollagen1, alpha smooth muscle actin, TNF-alpha, MCP-1, and ym-1 did not change between vehicle and VEGF-C groups (Supplementary Figure 2). We also speculate that the increased LVs did not affect the mRNA synthesis of fibrotic genes or proinflammatory cytokine genes, but they drained inflammatory cytokines, inflammatory cells, and interstitial fluid, which may be the reason why the mRNA levels did not change after VEGF-C administration.

Previous studies have shown that infiltration of F4/80positive macrophages increases in UUO kidneys, which is associated with increased LEC density and interstitial fibrosis. ${ }^{1,37,38}$ In our study, at around 14 days after the UUO operation, interstitial fibrosis was observed as secondary to inflammatory cell infiltration. Therefore, we administrated VEGF-C continuously for 14 days. As a result, F4/80-positive macrophages decreased in the VEGF-C group. This observation suggests that macrophages have a critical role in inflammation-induced interstitial fibrosis.

Fibrosis has several characteristic features including a chronic inflammatory reaction and increased interstitial fibroblast and matrix accumulation. ${ }^{37}$ The interstitial cellular response to UUO is associated with an increased inflammatory reaction..$^{37}$ Lymphangiogenesis is associated with acute or chronic inflammation. Therefore, tubular atrophy in UUO may be linked to lymphangiogenesis in the interstitial fibrotic area.

The important issue of lymphangiogenesis in renal fibrosis is whether it has a beneficial role in the destruction of renal parenchyma after ureteral obstruction. Recently, disturbance of lymphatic circulation by ligation was shown to induce renal fibrosis. Such changes in lymphatic flow could be a factor in renal fibrosis. ${ }^{17} \mathrm{~A}$ previous study demonstrated that fibrosis in soft tissue involves impairment of lymphatic cell proliferation and lymphatic functions, and suggested that the fibrotic process decreases lymphatic repair and regeneration of normal capillary lymphatics. ${ }^{39}$

In lymphangiogenesis of kidney diseases, an increase in the number of LVs is found at the site of tubulointerstitial lesions, which correlates with fibrosis and inflammation. ${ }^{1}$ Kerjaschki et al. ${ }^{40}$ have demonstrated that lymphangiogenesis contributes to the export of inflammatory cell infiltrates in human transplants. However, there is a paucity of data on the functional role of lymphangiogenesis in renal fibrosis, and further studies are needed. We demonstrated that VEGF-C ameliorated cell viability and proliferation, and induced expression of adhesion molecule genes. These results suggested that systemic administration of VEGF-C in UUO mice increased the number of LECs and improved the functions of LECs. UUO kidneys have markedly increased interstitial pressure. Increased lymphatic functions may drain excess interstitial fluid and pressure to suppress inflammatory cell infiltration, inflammatory cytokines, and interstitial 
fibrosis. In polycystic kidney disease mice, Huang et al. ${ }^{41}$ demonstrated that systemic administration of VEGF-C increases the size and number of LVs, reduces interstitial M2 macrophage infiltration, and decreases the size and number of renal cysts. Lymphatic functions increased by VEGF-C may ameliorate interstitial fibrosis by reducing excess interstitial fluid, infiltration of inflammatory cells, and cytokines. A previous report showed that proteinuria triggers new LV formation in the kidney. ${ }^{42}$ Proteinuria is associated with the activation of proinflammatory molecular pathways in renal interstitium and directly involved in the pathogenesis of tubulointerstitial fibrosis. ${ }^{43}$ Based on our results, lymphangiogenesis caused by proteinuria may be related to the pathophysiology of renal inflammation and fibrosis. The UUO mouse is a non-proteinuria model. Thus, further study is needed in this regard. In addition, as demonstrated previously, VEGF-C/VEGFR-3-mediated modification of cutaneous lymphatic functions may regulate blood pressure by local organization of interstitial electrolyte clearance via tonicity-responsive element-binding protein. ${ }^{5}$ In this model, administration of VEGF-C might also regulate blood pressure.

This study had several limitations. First, we could not confirm that inhibition of VEGFR-3 prevented the fibrosissuppressing effect of VEGF-C. Second, we investigated inflammation and fibrosis in the UUO model only at 14 days. Therefore, we could not establish the precise timing and action of VEGF-C in fibrosis and lymphangiogenesis. Third, we could not confirm the selectivity of human recombinant VEGF-C for mouse VEGFR-3 versus mouse VEGFR-2. However, we demonstrated increases in VEGFR-3 protein levels and VEGFR-3 phosphorylation, and no change in phospho-VEGFR-2 in the UUO+VEGF-C group. Finally, we could not evaluate lymphatic functions in detail, such as changes in lymphatic flow or substances that were drained. However, the information gained by our study contributes toward a better understanding of the pathogenesis of renal fibrosis and lymphangiogenesis in UUO mice.

In summary, our results suggest that VEGF-C has a role in suppression of interstitial inflammation in UUO mice. Suppression of inflammation was associated with amelioration of interstitial fibrosis. These findings suggest that lymphangiogenesis induced by VEGF-C may be a new therapeutic target to improve renal interstitial inflammation and fibrosis.

Supplementary Information accompanies the paper on the Laboratory Investigation website (http://www.laboratoryinvestigation.org)

\section{ACKNOWLEDGMENTS}

We thank Ms Hideko Noguchi for her excellent technical assistance. We appreciate the technical assistance from The Research Support Center, Research Center for Human Disease Modeling, and Kyushu University Graduate School of Medical Sciences. We also thank Edanz Editing (http:// www.edanzediting.co.jp/) for their English editing of our manuscript. This work was supported in part by JSPS KAKENHI Grants-in-Aid for Scientific Research C (No. 20590342 and 23590400).

\section{DISCLOSURE/CONFLICT OF INTEREST}

The authors declare no conflict of interest.

1. Sakamoto I, Ito $Y$, Mizuno $M$, et al. Lymphatic vessels develop during tubulointerstitial fibrosis. Kidney Int 2009;75:828-838.

2. Huggenberger R, Siddiqui SS, Brander D, et al. An important role of lymphatic vessel activation in limiting acute inflammation. Blood 2011;117:4667-4678.

3. D'Alessio S, Correale C, Tacconi C, et al. VEGF-C-dependent stimulation of lymphatic function ameliorates experimental inflammatory bowel disease. J Clin Invest 2014;124:3863-3878.

4. Nurmi H, Saharinen P, Zarkada G, et al. VEGF-C is required for intestinal lymphatic vessel maintenance and lipid absorption. EMBO Mol Med 2015;7:1418-1425.

5. Wiig H, Schroder A, Neuhofer W, et al. Immune cells control skin lymphatic electrolyte homeostasis and blood pressure. J Clin Invest 2013;123:2803-2815.

6. Tammela T, Alitalo K. Lymphangiogenesis: molecular mechanisms and future promise. Cell 2010;140:460-476.

7. Meinecke AK, Nagy N, Lago GD, et al. Aberrant mural cell recruitment to lymphatic vessels and impaired lymphatic drainage in a murine model of pulmonary fibrosis. Blood 2012;119:5931-5942.

8. Tsuchimoto A, Nakano $T$, Hasegawa $S$, et al. The potential role of perivascular lymphatic vessels in preservation of kidney allograft function. Clin Exp Nephrol 2016;21:721-731.

9. Veikkola T, Jussila L, Makinen T, et al. Signalling via vascular endothelial growth factor receptor-3 is sufficient for lymphangiogenesis in transgenic mice. EMBO J 2001;20:1223-1231.

10. Hamada K, Oike $\mathrm{Y}$, Takakura N, et al. VEGF-C signaling pathways through VEGFR-2 and VEGFR-3 in vasculoangiogenesis and hematopoiesis. Blood 2000;96:3793-3800.

11. Huggenberger R, Ullmann S, Proulx ST, et al. Stimulation of lymphangiogenesis via VEGFR-3 inhibits chronic skin inflammation. J Exp Med 2010;207:2255-2269.

12. Makinen T, Veikkola T, Mustjoki S, et al. Isolated lymphatic endothelial cells transduce growth, survival and migratory signals via the VEGF-C/D receptor VEGFR-3. EMBO J 2001;20:4762-4773.

13. Karkkainen MJ, Haiko $P$, Sainio $K$, et al. Vascular endothelial growth factor $C$ is required for sprouting of the first lymphatic vessels from embryonic veins. Nat Immunol 2004;5:74-80.

14. Kaipainen A, Korhonen J, Mustonen T, et al. Expression of the fms-like tyrosine kinase 4 gene becomes restricted to lymphatic endothelium during development. Proc Natl Acad Sci USA 1995;92:3566-3570.

15. Onimaru M, Yonemitsu Y, Fujii T, et al. VEGF-C regulates lymphangiogenesis and capillary stability by regulation of PDGF-B. Am J Physiol Heart Circ Physiol 2009;297:H1685-H1696.

16. Aukland K, Bogusky RT, Renkin EM. Renal cortical interstitium and fluid absorption by peritubular capillaries. Am J Physiol 1994;266: F175-F184.

17. Zhang T, Guan G, Liu G, et al. Disturbance of lymph circulation develops renal fibrosis in rats with or without contralateral nephrectomy. Nephrology (Carlton) 2008;13:128-138.

18. Joukov V, Kumar V, Sorsa $T$, et al. A recombinant mutant vascular endothelial growth factor-C that has lost vascular endothelial growth factor receptor-2 binding, activation, and vascular permeability activities. J Biol Chem 1998;273:6599-6602.

19. Masuda Y, Shimizu A, Mori T, et al. Vascular endothelial growth factor enhances glomerular capillary repair and accelerates resolution of experimentally induced glomerulonephritis. Am J Pathol 2001;159: 599-608.

20. Benedito $R$, Rocha SF, Woeste $M$, et al. Notch-dependent VEGFR3 upregulation allows angiogenesis without VEGF-VEGFR2 signalling. Nature 2012;484:110-114.

21. Ikeda H, Tsuruya K, Toyonaga J, et al. Spironolactone suppresses inflammation and prevents L-NAME-induced renal injury in rats. Kidney Int 2009;75:147-155.

22. Nakano T, Nakashima $Y$, Yonemitsu $Y$, et al. Angiogenesis and lymphangiogenesis and expression of lymphangiogenic factors in 
the atherosclerotic intima of human coronary arteries. Hum Pathol 2005;36:330-340.

23. Alitalo K, Tammela T, Petrova TV. Lymphangiogenesis in development and human disease. Nature 2005;438:946-953.

24. Suzuki $Y$, Ito $Y$, Mizuno $M$, et al. Transforming growth factor-beta induces vascular endothelial growth factor-C expression leading to lymphangiogenesis in rat unilateral ureteral obstruction. Kidney Int 2012;81:865-879.

25. Kunstfeld R, Hirakawa S, Hong YK, et al. Induction of cutaneous delayed-type hypersensitivity reactions in VEGF-A transgenic mice results in chronic skin inflammation associated with persistent lymphatic hyperplasia. Blood 2004;104:1048-1057.

26. Baluk $\mathrm{P}$, Tammela $\mathrm{T}$, Ator $\mathrm{E}$, et al. Pathogenesis of persistent lymphatic vessel hyperplasia in chronic airway inflammation. J Clin Invest 2005;115:247-257.

27. Pedica F, Ligorio C, Tonelli $\mathrm{P}$, et al. Lymphangiogenesis in Crohn's disease: an immunohistochemical study using monoclonal antibody D2-40. Virchows Arch 2008;452:57-63.

28. Geleff S, Schoppmann SF, Oberhuber G. Increase in podoplaninexpressing intestinal lymphatic vessels in inflammatory bowel disease. Virchows Arch 2003:442:231-237.

29. Alitalo K. The lymphatic vasculature in disease. Nat Med 2011;17: 1371-1380.

30. Zhang Q, Lu Y, Proulx ST, et al. Increased lymphangiogenesis in joints of mice with inflammatory arthritis. Arthritis Res Ther 2007;9:R118.

31. Saaristo A, Tammela T, Farkkila A, et al. Vascular endothelial growth factor-C accelerates diabetic wound healing. Am J Pathol 2006;169: 1080-1087.

32. Zheng $\mathrm{Y}$, Lin $\mathrm{H}$, Ling $\mathrm{S}$. Clinicopathological correlation analysis of (lymph) angiogenesis and corneal graft rejection. Mol Vis 2011;17: 1694-1700.

33. Kim H, Kataru RP, Koh GY. Regulation and implications of inflammatory lymphangiogenesis. Trends Immunol 2012;33:350-356.
34. Yin $\mathrm{N}$, Zhang $\mathrm{N}$, Xu J, et al. Targeting lymphangiogenesis after islet transplantation prolongs islet allograft survival. Transplantation 2011;92:25-30.

35. Dietrich T, Bock F, Yuen D, et al. Cutting edge: lymphatic vessels, not blood vessels, primarily mediate immune rejections after transplantation. J Immunol 2010;184:535-539.

36. Lee AS, Lee JE, Jung YJ, et al. Vascular endothelial growth factor-C and -D are involved in lymphangiogenesis in mouse unilateral ureteral obstruction. Kidney Int 2013;83:50-62.

37. Kim W, Moon SO, Lee SY, et al. COMP-angiopoietin-1 ameliorates renal fibrosis in a unilateral ureteral obstruction model. J Am Soc Nephrol 2006;17:2474-2483.

38. Naruse T, Yuzawa Y, Akahori T, et al. P-selectin-dependent macrophage migration into the tubulointerstitium in unilateral ureteral obstruction. Kidney Int 2002;62:94-105.

39. Avraham T, Clavin NW, Daluvoy SV, et al. Fibrosis is a key inhibitor of lymphatic regeneration. Plast Reconstr Surg 2009;124: 438-450.

40. Kerjaschki D, Regele HM, Moosberger I, et al. Lymphatic neoangiogenesis in human kidney transplants is associated with immunologically active lymphocytic infiltrates. J Am Soc Nephrol 2004;15:603-612.

41. Huang JL, Woolf AS, Kolatsi-Joannou M et al. Vascular endothelial growth factor C for polycystic kidney diseases. J Am Soc Nephrol 2016;27:69-77.

42. Yazdani $S$, Poosti $F$, Kramer $A B$, et al. Proteinuria triggers renal lymphangiogenesis prior to the development of interstitial fibrosis. PLoS ONE 2012;7:e50209.

43. Abbate M, Zoja C, Remuzzi G. How does proteinuria cause progressive renal damage? J Am Soc Nephrol 2006;17:2974-2984.

44. Pajusola K, Aprelikova O, Pelicci G, et al. Signalling properties of FLT4, a proteolytically processed receptor tyrosine kinase related to two VEGF receptors. Oncogene 1994;12:3545-3555. 